\title{
Building Bridges in Child and Adolescent Psychiatry Training: Providing Maternal-Infant Mental Health Care Through Creation of a Perinatal Collaborative Care Program
}

\author{
Misty C. Richards ${ }^{1}$ (1)
}

Received: 29 July 2021 / Accepted: 8 November 2021 / Published online: 29 November 2021

(c) Academic Psychiatry 2021

\section{To the Editor}

:Along with the birth of a child comes the birth of a mother, each unsteady in their literal and figurative gait. As mothers attempt to recover from pregnancy and labor, they enter a maternal marathon of sorts, attending to the unrelenting needs of an infant. Likewise, this fragile infant is learning how to communicate with the world and working very hard to grow and meet developmental milestones. This vulnerable window presents an invaluable opportunity for child and adolescent psychiatry fellows to learn a dyadic approach to mental health care with the ultimate goal of early intervention.

Infant mental health care does not exist without treating the caregiver, and the consequences of a one-sided approach can be catastrophic for a family. Mothers often suffer in silence with perinatal mood disorders, anxiety, and trauma, which can have significant downstream consequences for child development and psychopathology [1]. Children of depressed parents show greater vulnerability to psychopathology, particularly to depression, anxiety disorders, and maladaptive social behavior [2]. Evidence shows that the transmission of risk from depressed mothers to their children involves both genetic influences and detached parenting, the latter negatively effecting the attachment relationship [2]. In order to optimize the health of a child, providers must invest in the mental health of mothers at the earliest timepoint possible. This early intervention, especially with rates of perinatal depression estimated at $20 \%$, can prevent harmful and potentially traumatic narratives from being built and lay the foundation for secure attachment.

Unfortunately, providing quality mental health services to the mother-infant dyad continues to be a challenge, with

Misty C. Richards

mcrichards@mednet.ucla.edu

1 University of California, Los Angeles, CA, USA access to affordable, timely care serving as the major issue. This gap in patient care also represents a missed training opportunity for child and adolescent psychiatry trainees, who are well positioned to treat this vulnerable population. At UCLA, we have used this clinical barrier to fuel the creation of the Maternal Outpatient Mental health Services (MOMS) Clinic, which utilizes a perinatal collaborative care model between psychiatry and obstetrics supported by the Department of Obstetrics and Gynecology. The clinic has existed for two years, with preliminary data supporting the evidence-based benefits of collaborative care including improved access to behavioral health services, emphasis on a patient-centered approach, and coordination of care that facilitates improved patient outcomes [3]. The MOMS Clinic has trained several child and adolescent psychiatry trainees to provide holistic, dyadic mental health care with the intention of attenuating intergenerational trauma and setting children up for success from the very beginning.

The clinic emerged in 2019, after the release of Assembly Bill (AB) 2193 that required licensed perinatal health care practitioners in California to screen mothers for maternal mental health conditions. Subsequently, the OB-GYN Department screened every eligible woman with the Edinburgh Postnatal Depression Scale (EPDS), creating a steady referral stream to the MOMS Clinic [4]. The Department of OB-GYN and the Division of Child and Adolescent Psychiatry championed the MOMS Clinic, understanding that this investment would not only help with patient care but support training in both reproductive psychiatry and infant mental health. For child and adolescent psychiatry fellows, the MOMS Clinic offers a rare opportunity to work in an interdisciplinary team setting at the earliest intervention point in childhood. This exposure to infant mental health care emphasizes the importance of the dyad, teaching trainees to approach treatment through a developmental, attachment-based lens. In the MOMS Clinic, child and adolescent psychiatry fellows have the unique opportunity to provide 
patient care that utilizes a collaborate care model; observe reflective Circle of Security Parenting groups that provide a visual map of attachment; facilitate process groups; provide short-term, individual therapy; practice complex psychopharmacology; and observe the strength-based Newborn Behavioral Observation (NBO) Assessment, all while learning to weave psychoeducation into each of these services. This comprehensive training approach during the perinatal period is relatively rare for child and adolescent psychiatry fellows, building bridges between mother and infant, obstetrics and child psychiatry, and, perhaps most pressing, vulnerable populations and access to quality mental health care.

An unfortunate impact of the coronavirus disease 2019 (COVID-19) pandemic was the loss of psychosocial supports for new mothers, likely contributing to a $30 \%$ increase in patient volume to the MOMS Clinic [5]. In response, the MOMS Clinic converted to telehealth which resulted in an almost non-existent no-show rate and an efficient, predictable system allowing more families to be treated. Ultimately, telehealth facilitated broader access to our clinic, especially for those who have difficulty traveling to brick-and-mortar clinics, while normalizing a mother and infant's need for the comforts and safety of home. From an educational standpoint, this rapid transition to telehealth provided opportunities for trainees to master a new health care delivery tool, one that has preserved quality psychiatric care for 1.5 years now.

The MOMS Clinic has become a staple in the UCLA perinatal community. The OB-GYN and child psychiatry stakeholders are in place, the interdisciplinary team has been assembled, and the communication between psychiatrists and obstetricians is robust. Referrals to the clinic are steady, with warm handoffs made to community providers once mothers and infants are stabilized in the MOMS Clinic. The UCLA Child and Adolescent Psychiatry Fellowship endorses this clinic as a unique and rich training experience that requires fellows to utilize their clinical, developmental, and population health expertise. The educational objectives for child psychiatry trainees who rotate through the clinic are to learn about perinatal collaborative care, the importance of a dyadic approach to maternal-infant mental health care, and to bear witness to the recovery that is possible when dedicated medical disciplines join forces to care for patients. The training program intends to create a Maternal-Infant Mental Health Area of Distinction (AOD) in 2022 that will incorporate clinical opportunities provided by the MOMS Clinic coupled with a rich curriculum combining the latest evidence-base for both infant and perinatal mental health. Through knocking down barriers in training and building bridges across disciplines, we are confident that our unique training experience for child and adolescent psychiatry trainees will encourage early intervention that can make all the difference for vulnerable families.

\section{Declarations}

Disclosure The author states that there is no conflict of interest.

\section{References}

1. Muzik M, Hamilton SE. Use of antidepressants during pregnancy?: What to consider when weighing treatment with antidepressants against untreated depression. Matern Child Health J. 2016;20:2268-79.

2. Goodman SH. Depression in mothers. Annu Rev Clin Psychol. 2007;3:107-35.

3. Miller ES, Jensen R, Hoffman MC, Osborne LM, McEvoy K, Grote N, et al. Implementation of perinatal collaborative care: a health services approach to perinatal depression care. Prim Health Care Res Dev. 2020;21:1-9.

4. Cox JL, Holden JM, Sagovsky R. Detection of postnatal depression. Development of the 10-item Edinburgh Postnatal Depression Scale. Br J Psychiatry. 1987;150:782-6.

5. Hermann A, Fitelson EM, Bergink V. Meeting maternal mental health needs during the COVID-19 pandemic. JAMA Psychiat. 2021;78:123-4.

Publisher's Note Springer Nature remains neutral with regard to jurisdictional claims in published maps and institutional affiliations. 